\title{
Effect of the Daily Ingestion of a Purified Anthocyanin Extract From Grape Skin on Rat Serum Antioxidant Capacity
}

\author{
M. G. LIONETTO ${ }^{1}$, M. E. GIORDANO ${ }^{1}$, A. CALISI ${ }^{1}$, E. ERROI ${ }^{1}$, F. DE NUCCIO ${ }^{1}$, \\ T. SCHETTINO ${ }^{1}$ \\ ${ }^{1}$ Department of Biological and Environmental Sciences and Technologies, University of Salento, \\ Italy
}

Received July 24, 2010

Accepted December 10, 2010

On-line May 16, 2011

\section{Summary}

The aim of this work was to study the effect of the daily ingestion of a purified anthocyanin extract from red grape skin on rat serum antioxidant capacity (ORAC) and its safety for the intestinal epithelium. The study was carried out in rats orally administered with the extract for 10 days in either normal physiological conditions or exposed to a pro-oxidant chemical $\left(\mathrm{CCl}_{4}\right)$. The oral administration of the extract significantly $(P<0.05)$ enhanced the ORAC value of the deproteinised serum of about $50 \%$ after 10 days of ingestion. Anthocyanin administration was also able to reverse completely the decrease in the serum ORAC activity induced by the $\mathrm{CCl}_{4}$ treatment. Experiments with Ussing chamber mounted intestine allowed to exclude any toxicity of the extract for the intestinal epithelium. In conclusion, our results demonstrate that the purified anthocyanin extract from red grape skin enhances the total antioxidant capacity of the serum in either normal physiological condition or during oxidative stress induction, revealing a protective role against the decrease in the serum antioxidant capacity induced by a pro-oxidant compound.

\section{Key words}

Anthocyanin • Antioxidant capacity • ORAC • Grape skin • Serum

\section{Corresponding author}

M. G. Lionetto, Dipartamento to di Scienze e Tecnologie Biologiche e Ambientali, Università di Lecce, Via provinciale Lecce-Monteroni, 73100 Lecce, Italy. Fax +390832 298626. E-mail: giulia.lionetto@unisalento.it

\section{Introduction}

Anthocyanins are chemically phenolic compounds belonging to the flavonoid family responsible for the colours of flowers and fruits of a great variety of plants. They have been shown to possess beneficial in vitro properties related to the protection against pathologies involving oxidative stress, such as cardiovascular diseases, cancer, neurodegeneration, inflammation, and viral infection (Prior and Wu 2006). In particular, anthocyanins are potent antioxidants (Khkonen and Heinonen 2003) because of their ability to quickly reduce oxidizing species while being converted into stabilized aryloxyl radicals. Moreover, they are able to chelate transition metal ions potentially involved in the development of oxidative stress (Moran et al. 1997).

In grapes (Vitis vinifera, L.) anthocyanins are the typical pigments of the skins of red cultivars, therefore grape skins represent an excellent source of anthocyanins. The anthocyanins identified in Vitis vinifera spp. correspond to the 3-O-monoglucosides and the 3-Oacylated monoglucosides of the five main anthocyanidins: delphinidin, cyanidin, petunidin, peonidin, and malvidin. The antioxidant activity of red grape anthocyanins has been extensively demonstrated mainly in in vitro studies. Frankel et al. (1998) demonstrated the in vitro inhibition of the copper catalyzed oxidation of human LDL by red commercial grape juices and related this activity to the anthocyanin concentrations. Singletary et al. (2007) demonstrated the anthocyanins contained in a red grape extract to have in vitro breast cancer chemopreventive potential due in part 
to their capacity to block carcinogen-DNA adduct formation, to modulate activities of carcinogenmetabolizing enzymes, and antioxidant activity. Zenebe et al. (2003) demonstrated red wine polyphenols, including anthocyanin, to induce vasorelaxation by increased nitric oxide bioactivity.

Extracts from Vitis vinifera are commonly used to formulate dietary antioxidant supplements. Although the dietary industry based on wine by-products is rapidly growing, practically no scientific research is available on the in vivo antioxidant activity of these products and their potential effects and safety for the gastrointestinal tract.

The aim of this work was to study the in vitro and in vivo antioxidant activity and the safety for the intestinal epithelium of a purified anthocyanin extract, obtained from red grape skin by an innovative method based on supercritic $\mathrm{CO}_{2}$ (Bleve et al. 2008) and available to be used for dietary antioxidant supplement. As previously demonstrated (Bleve et al. 2008), this purification method allowed to eliminate the solvent residue from the anthocyanin extract without any thermal or chemical degradation, obtaining a high added value product which is unchanged in its anthocyanin content. According to Bleve et al. (2008), four major anthocyanins were present in the purified extract: malvidin 3-O-glucoside (50\%), petunidin 3-O-glucoside (10\%), delphinidin 3-O-glucoside (8\%), and cyanidin 3-O-rutinoside ( $5 \%)$.

In the present paper the in vitro and in vivo antioxidant activity of the anthocyanin extract was determined by the ORAC (Oxygen Radical Absorbance Capacity) assay which is still one of the few methods that combines both inhibition percentage and inhibition time of the reactive species action by antioxidants in a single quantity.

The in vivo antioxidant properties of the extract were studied by measuring the total antioxidant capacity (ORAC) of the deproteinised serum of rats orally administrated with the extract for ten days. The total antioxidant capacity of blood plasma or serum by the ORAC method represents an integrated measure of the non enzymatic antioxidant defence mechanisms of the organism (Cao et al. 1998). The effects of the anthocyanin extract ingestion were analyzed in either normal physiological conditions or after treatment with a single dose of $\mathrm{CCl}_{4}$ in order to investigate the possible protective action of the extract administration against oxidative stress induced by the exposure to a pro-oxidant agent. $\mathrm{CCl}_{4}$ is a well established hepatotoxin (Szymonik-
Lesiuk et al. 2003). However, various studies demonstrated that liver is not the only target organ of $\mathrm{CCl}_{4}$ and that this compound causes free radical generation also in other tissues such as kidneys, heart, lung, testis, brain, and blood (Ohta et al. 1997, Ozturk et al. 2003).

The potential effect of the extract on intestinal cells functionality was assessed in vitro, by exposing the jejunum tract of rat intestine to the extract and monitoring transepithelial electric resistance $\left(\mathrm{R}_{\mathrm{te}}\right)$ and lactate dehydrogenase (LHD) release.

The results give more insight on the in vivo antioxidant activity and safety for the gastrointestinal tract of a purified red grape anthocyanin extract, and point out the protective effect of the prolonged daily ingestion of anthocyanins on the serum antioxidant capacity observed during exposure to a pro-oxidant compound.

\section{Materials}

The anthocyanin purified extract was provided by Pierre Chimica S.r.l., Galatina, Lecce, Italy and Dip. of Innovation Engineering, University of Salento Lecce, Italy. According to Bleve et al. (2008) four major anthocyanins were present in the extract: malvidin 3-O-glucoside $(50 \%)$ petunidin 3 - $O$-glucoside $(10 \%)$, delphinidin 3-O-glucoside $(8 \%)$, and cyanidin 3 -O-rutinoside $(5 \%)$. $0.16 \mathrm{~g}$ of extract were obtained per one gram of grape skin wet weight.

Male Wistar rats weighing 200 to $250 \mathrm{~g}$ were purchased from Harlan Italy s.r.l. (UD, Italy). All chemicals were reagent grade and were purchased from Sigma (St. Louis, U.S.A.).

\section{Methods}

All experiments were carried out in accordance with the European Guidelines on Laboratory Animal Care and had the approval by the Italian Ministry of Health.

\section{ORAC assay of the anthocyanin extract}

The ORAC protocol utilized in this work refers to the method described by Ou et al. (2001) and further modified by Davalos et al. (2004) which uses fluorescein instead of $\beta$-phycoerythrin as fluorescent probe. Briefly, the diluted anthocyanin-rich extract $(20 \mu \mathrm{l})$ and the fluorescein solution (1200 $\mu 1,120 \mathrm{nM}$ in phosphate buffer, $\mathrm{pH}$ 7.4) were pre-incubated for $15 \mathrm{~min}$ at $37^{\circ} \mathrm{C}$; 
then the peroxyl radical generator AAPH $(600 \mu 1,40 \mathrm{mM}$ in phosphate buffer, $\mathrm{pH}$ 7.4) was added. The fluorescence of the mixture was recorded every minute for $80 \mathrm{~min}$ $\left(\lambda_{\mathrm{ex}}=535 ; \lambda_{\mathrm{em}}=560 \mathrm{~nm}\right)$. Trolox $(1,2,4$ and $8 \mu \mathrm{M}$ final concentration) was used as antioxidant standard for the calibration curve construction. For each fluorescent decay curve (fluorescence versus time) (Fig. 1) the area under curve (AUC) was calculated as follows:

$$
\mathrm{AUC}=\sum_{\mathrm{i}=0}^{\mathrm{n}} \mathrm{f}_{\mathrm{i}} / \mathrm{f}_{0}
$$

where $f_{0}$ is the initial fluorescence reading at $0 \mathrm{~min}$ and $f i$ is the fluorescence reading at time $i$. For each sample the net AUC was calculated by subtracting the AUC corresponding to the blank (fluorescein + AAPH without the antioxidant solution). The AUC value was interpolated on the calibration curve. The ORAC values were expressed as micromoles of trolox equivalents.

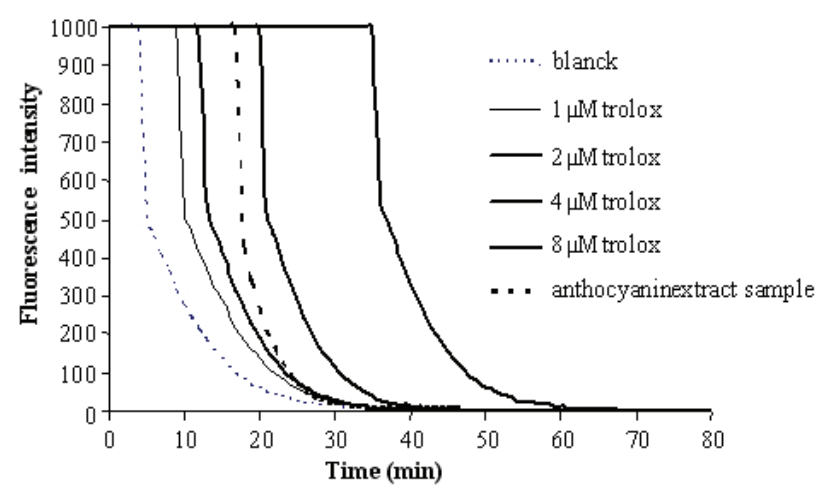

Fig. 1. Fluorescent decay curves of trolox $(1 \mu \mathrm{M}, 2 \mu \mathrm{M}, 4 \mu \mathrm{M}$, $8 \mu \mathrm{M})$, utilized as standard, and anthocyanin extract sample. Representative determination.

\section{Experimental design of in vivo rat exposure}

Male Wistar rats weighing 200 to $250 \mathrm{~g}$ were kept in a temperature- and humidity-controlled room with 12-hour light/dark cycles. They were given free access to tap water and standard rat chow.

In the first exposure experiment 16 rats were divided in two groups: 1) control animals (not exposed to any treatment); 2) anthocyanin exposed animals, orally administrated by micropipette to an anthocyanin extract solution at the dosage of $0.6 \mathrm{mg}$ of anthocyanin per $\mathrm{kg}$ of body weight. The animals were daily exposed to the extract for ten days in order to experimentally simulate a prolonged exposure. Thereafter, they were sacrificed at the $11^{\text {th }}$ day of the experiment.

In the second exposure experiment 32 rats were divided into four groups (eight animals for each group) composed as follows: 1) control animals; 2) animals daily exposed for ten days by oral administration to the anthocyanin extract (as reported above); 3) animals who received one dose of a subcutaneous injection of $\mathrm{CCl}_{4}$ at the eighth day of the experiment; 4) animals daily exposed to the extract as above who received in addition one dose of a subcutaneous injection of $\mathrm{CCl}_{4}$ at the eighth day of the experiment. Control and anthocyanin exposed animals received one dose of a subcutaneous injection of oil (vehicle of $\mathrm{CCl}_{4}$ ) at the eighth day of the experiment. According to Tirkey et al. (2005), $\mathrm{CCl}_{4}$ was applied only at $8^{\text {th }}$ day of the experiment in order to reduce the risk of mortality following a prolonged exposure to the drug.

For the second exposure experiment an orthogonal two factor experimental design was chosen: factor (A) "anthocyanin exposure" which included two levels ("not exposed" and "exposed"), and factor (B) " $\mathrm{CCl}_{4}$ exposure" which included two levels ("not exposed" and "exposed).

\section{Blood sample preparation and ORAC assay}

At the $11^{\text {th }}$ day of the experiment the animals were anaesthetized with chloroform prior to sampling and then killed by decapitation. Blood was collected in a micro-tube within $5 \mathrm{sec}$ after decapitation. Then, the blood was allowed to clot at room temperature for $30 \mathrm{~min}$. The clot was removed by centrifuging at 2,000 $\mathrm{g}$ for 10 minutes in a refrigerated centrifuge and the resulting supernatant was collected. The serum was immediately diluted with saturated ammonium sulfate $(1: 4 \mathrm{v} / \mathrm{v})$, incubated for $10 \mathrm{~min}$ and then centrifuged at $11.500 \mathrm{rpm}$ for $30 \mathrm{~min}$ at $4{ }^{\circ} \mathrm{C}$. The supernatant was removed as deproteinized fraction that preserves the water-soluble antioxidants within the sample. The supernatant was utilized for the ORAC assay, as above described.

Tests on rat intestine: $R_{\text {te }}$ measurements and $L D H$ release assay

With the aim of evaluating the potential effects of the anthocyanin extract on the intestinal mucosa functionality, segments of rat jejunum intestine were placed in direct contact with the extract for a 3-hour incubation time. The epithelial barrier integrity was monitored by means of transepithelial electric resistance $\left(\mathrm{R}_{\mathrm{te}}\right)$ measurements. The LDH release was measured during and after the exposure respectively, as significant indicator of cell damage (Sannino et al. 2006). 3-hour 
incubation time is expected to approximate the real time of contact in vivo where food passes through the duodenum and jejunum. The jejunum represents the gastrointestinal segment where most digestion and absorption occurs.

The experiments were carried out on native tissues mounted in an Ussing chamber set up (Clarke 2009). This experimental approach has advantages in surveillance of the mucosa functionality compared to other in vitro techniques (Smith 1996).

Four male Wistar rats (weighing about $250 \mathrm{~g}$ ) were sacrificed for the experiment. Two adjacent segments $(1.5 \mathrm{~cm}$ length) of the jejunum tract were isolated from each animal, cut along the intestine longitudinal axis to produce a mucosal sheet and mounted vertically in an Ussing chamber. After mounting, each half chamber was filled with $6 \mathrm{ml}$ Krebs-buffer (containing in $\mathrm{mM}: \mathrm{NaCl} 124, \mathrm{KH}_{2} \mathrm{PO}_{4} 1.25, \mathrm{MgCl}_{2} 1.8$, $\mathrm{KCl} 1.75, \mathrm{CaCl}_{2}$ 1.6, $\mathrm{NaHCO}_{3} 26$, glucose 10), bathing the intestinal tissue on both the mucosal and serosal side. The exposed tissue surface area was $0.6 \mathrm{~cm}^{2}$. The Krebsbuffer was continuously oxygenated with $\mathrm{O}_{2} / \mathrm{CO}_{2}$ $(95 / 5 \%)$ and stirred by gas flow in the chambers. The equilibrium between $\mathrm{CO}_{2}$ and $\mathrm{NaHCO}_{3}$ maintained the $\mathrm{pH}$ of the Krebs buffer at the constant value of 7.4. The temperature of the Krebs solution was kept constant at $28^{\circ} \mathrm{C}$ all through the experiment. Tissues were connected to an automatic short-circuit current device (WPI's DVC-1000) by four $\mathrm{Ag} / \mathrm{AgCl}$ electrodes which made contact with the bathing solutions via agar-Ringer filled cartridges. Transepithelial voltage $\left(\mathrm{V}_{\mathrm{te}}\right)$ was measured with respect to the mucosal bath (grounded); the short circuit current $\left(\mathrm{I}_{\mathrm{sc}}\right)$ was measured by passage of sufficient current through $\mathrm{Ag} / \mathrm{AgCl}$ electrodes to reduce the spontaneous $\mathrm{V}_{\text {te }}$ to zero automatically (the resistance of the chamber fluid was subtracted automatically). $\mathrm{R}_{\mathrm{te}}$ was measured by pulsed current injection $\left(20 \mu \mathrm{A} \cdot \mathrm{cm}^{-2}\right.$, $500 \mathrm{~ms}$ ) through the tissue. The resulting $\mathrm{V}_{\text {te }}$ deflection was measured.

For each experiment the two adjacent jejunum segments were treated as follows: segment (1) was taken as a control, i.e. simply bathed with physiological solution, segment (2) was exposed to the extract $(0.02 \mathrm{mg} / \mathrm{ml}$ final concentration) from the luminal side of the epithelium. $V_{\text {te }}$ and $I_{s c}$, which are expression of the ion transport function of the intestine, were constantly monitored as tissutal viability index. All through the experiments $V_{\text {te }}$ and $I_{s c}$ showed the constant value of $1.5 \pm 0.2 \mathrm{mV}$ and $25 \pm 2.1 \mu \mathrm{A} \cdot \mathrm{cm}^{-2}$ respectively, which are typical for this tract of rat intestine (Madsen et al. 1996).

At the end of the experiment, the lactate dehydrogenase (LDH) leakage into the mucosal and serosal bathing solutions was monitored. LHD activity was measured according to Wroblewski and La Due (1955) and was expressed as Enzymatic Units per milliliter $\left(\mathrm{Uml}^{-1}\right)$. Triplicate determinations were performed for each sample.

\section{Statistical analysis}

Data were statistically analyzed by Student $t$ test (for the first anthocyanin in vivo exposure experiment) and by two ways ANOVA and Newman-Keuls post test (for the second in vivo exposure experiment). The homogeneity of variance was tested by Cochran's test prior to applying ANOVA. Data are reported as means \pm S.E.M.

\section{Results}

\section{In vitro ORAC activity of the purified extract}

The in vitro antioxidant activity of the purified anthocyanin extract, as determined by the ORAC assay, was $370 \pm 18.3$ ORAC per gram of grapes skin wet weight. When the antioxidant activity was referred to the anthocyanin concentration of the extract, it corresponded to $355 \pm 17.5$ ORAC/mg anthocyanin. The samples showed good antioxidant properties in terms of in vitro peroxyl radical scavenging activities compared to other grape skin extracts reported in literature (Yilmaz and Toledo 2003).

Effect of the anthocyanin administration on rat serum antioxidant capacity

The total antioxidant capacity of blood plasma is mainly derived from small molecules such as ascorbic acid, $\alpha$-tocopherol, $\beta$-carotene, and from macromolecules such as transferrin and ceruloplasmin. In order to better investigate the contribution of anthocyanin ingestion to the serum antioxidant capacity, the protein contribution to the total antioxidant capacity was excluded by performing the ORAC measurement on a serum deproteinized fraction.

With the aim to evaluate the potential effect of the prolonged daily ingestion of anthocyanins on the total serum antioxidant capacity, the rats were orally administrated with the purified extract for ten days. The daily intake of red grape anthocyanins utilized in the present work $(0.6 \mathrm{mg}$ of anthocyanin per $\mathrm{kg}$ of body 
weight) represents a fraction (about 20-25\%) of the total average daily intake of anthocyanins per $\mathrm{kg}$ of body weight in humans according to Kühnau (1976).

Table 1. Effect of the anthocyanin administration on rat serum antioxidant capacity. The Oxygen Radical Absorbance Capacity (ORAC) of deproteinized serum, expressed as ORAC (micromoles Trolox Equivalents)/ml of serum, was measured on control and anthocyanin administrated rats. The animals were daily exposed (by oral administration) to an extract amount corresponding to $0.6 \mathrm{mg}$ of anthocyanin per $\mathrm{kg}$ of body weight as described in the method section. $\mathrm{n}=$ number of animals. Data are reported as mean \pm S.E.M.* t-Student.

\begin{tabular}{lccc}
\hline & ORAC/ml & n & $\boldsymbol{P}^{*}$ \\
\hline Control rats & $0.574 \pm 0.083$ & 8 & \\
Anthocyanin & $0.883 \pm 0.089$ & 8 & 0.0236 \\
exposed rats & & & \\
\hline
\end{tabular}

As observed in Table 1 the rats orally administrated for ten days with the purified anthocyanin extract showed a significant increase in the antioxidant capacity of the deproteinized serum of about $50 \%$.

Effect of the anthocyanin administration on rat serum antioxidant capacity in pro-oxidant conditions

After having demonstrated the increase of rat serum antioxidant capacity following daily ingestion of the anthocyanin extract, we addressed the effect of the anthocyanin administration on serum ORAC in prooxidant conditions.

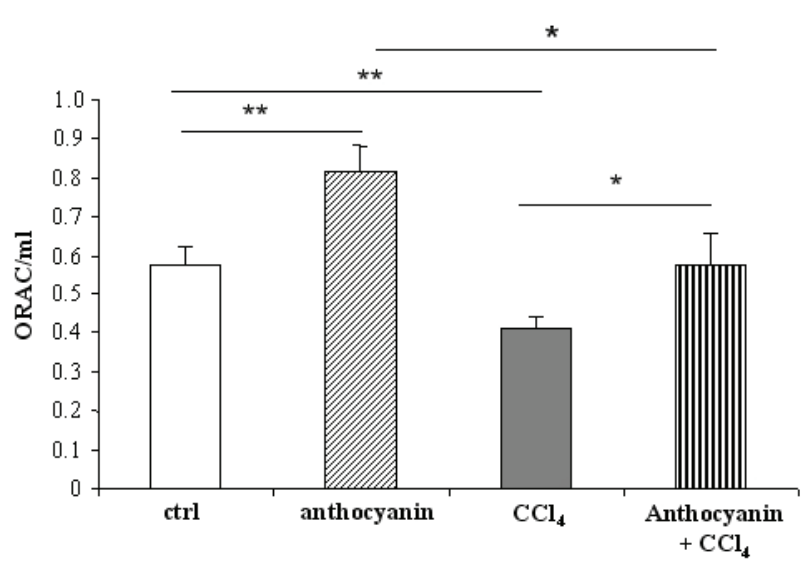

Fig. 2. Effect of the anthocyanin administration on rat serum antioxidant capacity in either physiological condition or after treatment with $\mathrm{CCl}_{4}$. The Oxygen Radical Absorbance Capacity (ORAC) of deproteinized serum was measured on: 1) control animals; 2) animals daily exposed to the anthocianin extract; 3 ) animals exposed to $\left.\mathrm{CCl}_{4} ; 4\right)$ animals exposed at first to anthocyanin and then to $\mathrm{CCl}_{4}$. Data are reported as mean \pm S.E.M. Data were analyzed by two way ANOVA and Newman Keules post test. * $\mathrm{P}<0.05 ; * * \mathrm{P}<0.01$.
When the animals were treated with the prooxidant agent $\mathrm{CCl}_{4}$, the serum antioxidant capacity significantly $(\mathrm{P}<0.01)$ decreased (Fig. 2). Interestingly, when the animals where orally administrated with the anthocyanin extract before treatment with $\mathrm{CCl}_{4}$, the effect of $\mathrm{CCl}_{4}$ was completely reversed resulting not statistically different from control animals (Fig. 2). Two way ANOVA analysis showed that either anthocyanin administration or $\mathrm{CCl}_{4}$ treatment had a significant $(P<0.01)$ effect on serum ORAC.

\section{$R_{\text {te }}$ measurement and LDH assay}

With the aim of evaluating the potential effects of the anthocyanin extract on the intestinal mucosa functionality, different segments of rat jejunum intestine were placed in direct contact with the extract for a 3-hour incubation time. The concentration utilized $(0.02 \mathrm{mg} / \mathrm{ml}$ final concentration) in vitro is consistent with the concentration used in in vivo extract oral administration and takes into account the dilution that the ingested substances undergo through the gut lumen. The gastrointestinal epithelium normally functions as a selective barrier that permits the absorption of nutrients, electrolytes, and water. The epithelial barrier function is provided by the epithelial cells and the tight junctions that connect them. The integrity of tight junctions is fundamental for the functional integrity of the tissue and can be electrophysiologically expressed by the $\mathrm{R}_{\mathrm{te}}$.

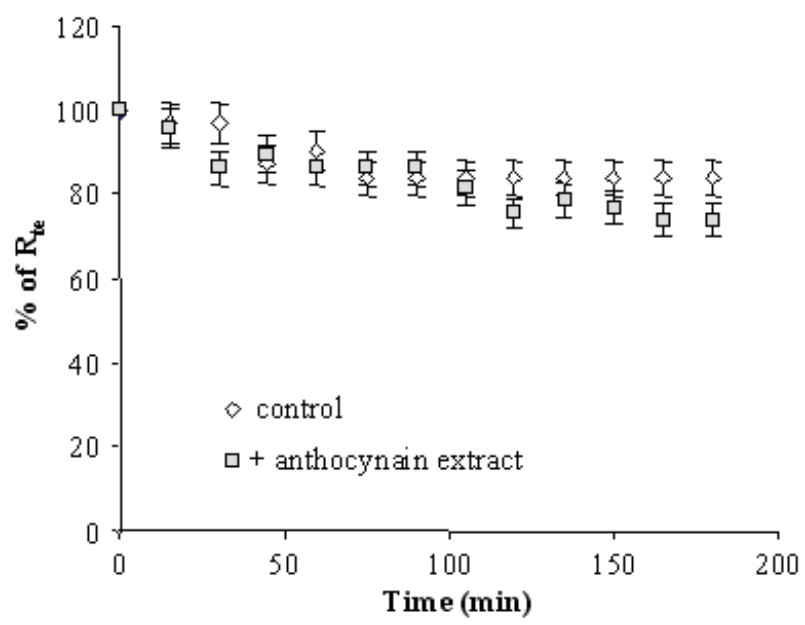

Fig. 3. Transepithelial electrical resistance $\left(R_{t e}\right)$ of rat jejunum, monitored during the exposure to the anthocyanin extract. Data are expressed as percentage of the initial value measured before the incubation with the extract. The time of contact of about 3 hours is expected to approximate the real time of contact in vivo. Data are reported as mean \pm S.E.M. of three independent experiments. 
In Figure $3 \mathrm{R}_{\mathrm{te}}$ values through the course of the experiment are reported as percentage of the initial value (corresponding to $20.2 \pm 1.2 \Omega \mathrm{cm}^{2}$ ), measured before the incubation of the epithelium with the extract. It can be observed that the exposure of the rat intestine with the extract does not significantly affect the $R_{t e}$ values, if compared to the control (segment perfused only with physiological solution). The statistical significance of the data was tested by Dunnett's test after transforming percentage data in corresponding arcsen values. These results suggest that the extract does not significantly affect the integrity of gastrointestinal epithelium or alter its permeability in in vitro conditions.

Table 2. Lactate dehydrogenase (LDH) release by rat intestinal cells after 3 hour incubation with the extract. The release of LDH has been measured both in the mucosal and serosal perfusates, as explained in the method section. The reported results are expressed as mean \pm S.E.M. $(n=3)$ of three independent experiments.

\section{Control +anthocyanin $(\mathrm{mU} / \mathrm{ml}) \quad$ extract \\ $(\mathbf{m U} / \mathbf{m l})$}

\begin{tabular}{lll} 
Mucosal solution & $5.90 \pm 1.11$ & $4.09 \pm 0.65$ \\
Serosal solution & $5.55 \pm 0.65$ & $4.40 \pm 1.10$ \\
\hline
\end{tabular}

Cytosolic LDH is widely used as a sensitive measure of cellular damage such as cell membrane injury (Whittle and Steel 2002). The results of LDH activity measured in the mucosal and serosal perfusates, collected after the 3-hour period of incubation of rat intestine, are reported in Table 2. No significant release of $\mathrm{LDH}$ is detected for the anthocyanin extract exposed segments either in the serosal or mucosal bathing solution with respect to the control tissues, as stated by Dunnett's test.

\section{Discussion}

In the present paper the in vivo effect of a purified anthocyanin extract from red grape skin on the total antioxidant capacity of deproteinised rat serum was demonstrated after a prolonged daily ingestion in either normal physiological conditions or after treatment with a single dose of $\mathrm{CCl}_{4}$. Experiments with Ussing chamber mounted intestine allowed to exclude any significantly toxic effect of the extract on the integrity and functionality of gastrointestinal epithelium of the experimental animals.

In normal physiological condition we demonstrated that the oral administration of the purified anthocyanin extract significantly $(\mathrm{P}<0.05)$ enhanced the oxygen radical absorbance capacity of the deproteinised serum of about $50 \%$ after 10 days of ingestion. It is known that the contribution of the non protein fraction to the antioxidant capacity of the serum is about $10-20 \%$ in adult rats (Cao et al. 1996), being higher in the newborn and decreasing significantly during growth. Therefore, according to Cao et al. (1996), the observed ORAC increase should correspond to an increase of the total antioxidant capacity of the entire serum of about 5-10\%. Increasing the serum antioxidant status has been implicated as a possible preventative means to reduce the development of cardiovascular disease (Kaplan and Aviram 1999, Salonen et al. 2000), diabetes (Vendemiale et al. 1999) and cancer (Willett 2001). Recently, researchers have determined a correlation between increased ex vivo blood antioxidant status and risk of cardiovascular disease and cancer (Durak et al. 2001, Ching et al. 2002).

It is known that after ingestion anthocyanins quickly appear as intact molecules in the plasma (Bub et al. 2001, Mazza et al. 2002, Wu et al. 2002). Therefore, it is possible to attribute the observed increase in the antioxidant activity to the increased anthocyanin concentration in the plasma. The use of a purified extract in our experiment allows to exclude the contribution of other antioxidants also present in the red grape skin (such as other polyphenols) to the observed serum antioxidant activity increase. Moreover, it allows also to exclude any interference with the plasma concentration of other antioxidants like the known effect of fructose on urate plasma concentration (Lotto and Frei 2004). In fact, it is known that the ingestion of fructose, often contained in many not purified anthocyanin extracts or in juices, can increase the plasma concentration of urate which is one of the main components of deproteinized serum antioxidant capacity (Lotto and Frei 2004). With respect to the oral intake of red grape juice reported in other works (Bitsch et al. 2004), the use of the purified anthocyanin extract in our experiments allows to ascribe the observed changes in the antioxidant capacity of plasma only to the anthocyanin contribution. In previous studies carried out on single anthocyanin administration, the anthocyanin plasma levels and the serum antioxidant activity returned to the basal level within two hours (Kay and Holub 2002, Bitsch et al. 2004). What is not known is if anthocyanins 
are accumulated in tissues when consumed over an extended period of time. In the present work the serum antioxidant capacity, measured at the $11^{\text {th }}$ day of experiment after a prolonged exposure to a purified anthocyanin extract for ten days, was still increased. This result seems to suggest a possible accumulation of anthocyanin in plasma when daily ingested for a prolonged period of time. However, this represents an intriguing hypothesis that need to be tested in a future work.

Moreover, in the present paper the in vivo effect of the purified anthocyanin extract from red grape skin on the total antioxidant capacity of the deproteinised serum was demonstrated after treatment with a pro-oxidant agent, $\mathrm{CCl}_{4}$. The hepatoxicity of $\mathrm{CCl}_{4}$ is well documented; however various studies demonstrated that liver is not the only target organ of $\mathrm{CCl}_{4}$ and that this compound causes free radical generation also in other tissues such as kidneys, heart, lung, testis, brain, and blood (Ohta et al. 1997, Ozturk et al. 2003). This compound generates in vivo trichloromethyl radicals that stimulate a sequence of reactions culminating in the initiation of the peroxidation of membrane lipids. It is known that $\mathrm{CCl}_{4}$ causes an elevation in the serum content of alanine transaminase and aspartate transaminase which is indicative of damage to the liver and other organs of the body (Reinke et al. 1988). It is also known that treatment of rats with anthocyanin for 4 weeks before $\mathrm{CCl}_{4}$ administration caused progressively less hepatotoxicity of $\mathrm{CCl}_{4}$ alone, as evidenced by the decreased serum content of alanine transaminase and aspartate transaminase relative to the $\mathrm{CCl}_{4}$-treated anthocyanin-free group (Obi et al. 1998). In the present paper for the first time a decrease of the serum oxygen radical absorbance capacity (ORAC) induced by $\mathrm{CCl}_{4}$ is reported. Surprisingly, when the animals were pretreated with the purified anthocyanin extract for ten days the inhibitory effect of $\mathrm{CCl}_{4}$ was completely reversed. Considering that the oxygen radical absorbance capacity (ORAC) measures the capacity of plasma/serum to inhibit peroxyl radical-induced oxidations (Cao et al. 1993), the obtained results suggest a protective role of the daily consumption of anthocyanin against the peroxyl radicals induced by $\mathrm{CCl}_{4}$. In general, peroxyl radicals are the predominant free radical found in biological systems (Prior et al. 2005) and the exposure to several agents, such as chemicals, drugs, and pollutants are known to induce peroxyl radicals in the organisms. Therefore, by extrapolation it is possible to argue a potential protective effect of the anthocyanin daily ingestion against oxidative stress induced by peroxyl radicals coming from different sources.

\section{Conclusions}

Our results demonstrate that the purified anthocyanin extract from red grape skin enhances the total antioxidant capacity of the serum in either normal physiological condition or during oxidative stress induction, revealing in this case a protective role against the decrease in the serum antioxidant capacity induced by a pro-oxidant compound. This work contributes to increase the knowledge of the effects of daily consumption of anthocyanin on the antioxidant defences of the organism.

\section{Conflict of Interest}

There is no conflict of interest.

\section{Acknowledgements}

This work has been financially supported by Fondazione Cassa di Risparmio di Puglia.

\section{References}

BITSCH R, NETZEL M, FRANK T, STRASS G, BITSCH I: Bioavailability and biokinetics of anthocyanins from red grape juice and red wine. J Biomed Biotechnol 5: 293-298, 2004.

BLEVE M, CIURLIA L, ERROI E, LIONETTO MG, LONGO L, RESCIO L, SCHETTINO T, VASAPOLLO G: An innovative method for the purification of anthocyanins from grape skin extracts by using liquid and sub-critical carbon dioxide. Sep Puri Technol 64: 192-197, 2008.

BUB A, WATZL B, HEEB D, RECHKEMMER G, BRIVIBA K: Malvidin-3-glucoside bioavailability in humans after ingestion of red wine, dealcoholized red wine and red grape juice. Eur J Nutr 40: 113-120, 2001.

CAO G, ALESSIO HM, CUTLER RG: Oxygen-radical absorbance capacity assay for antioxidants. Free Radic Biol Med 14: 303-311, 1993. 
CAO G, GIOVANONI M, PRIOR LM: Antioxidant capacity decreases during growth but not aging in rat serum and brain. Arch Geront Geriat 22: 27-37, 1996.

CAO G, RUSSEL RM, LISCHNER N, PRIOR RL: Serum antioxidant capacity is increased by consumption of strawberries, spinach, red wine or vitamin C in elderly women. J Nutr 128: 2383-2390, 1998.

CHING S, INGRAM D, HAHNEL R, BEILBY J, ROSSI E: Serum levels of micronutrients, antioxidants and total antioxidant status predict risk of breast cancer in a case control study. J Nutr 132: 303-306, 2002.

CLARKE LL: A guide to Ussing chamber studies of mouse intestine. Am J Physiol 296: G1151-G1166, 2009.

DÁVALOS A, GÓMEZ-CORDOVÉS C, BARTOLOMÉ B: Extending applicability of oxygen radical absorbance capacity (ORAC Fluorescein) assay. J Agric Food Chem 52: 48-54, 2004.

DURAK II, KACMAZ M, CIMEN MY, BUYUKKOCK U, OZTURK HS: Blood oxidant/antioxidant status of atherosclerotic patients. Int J Cardiol 77: 293-297, 2001.

FRANKEL EN, BOSANEK CA, MEYER AS, SILLIMAN K, KIRKS LL: Commercial grape juices inhibit the in vitro oxidation of human low-density lipoproteins. J Agric Food Chem 46: 834-838, 1998.

KAPLAN M, AVIRAM M: Oxidized low density lipoprotein: atherogenic and proinflammatory characteristics during macrophage foam cell formation. An inhibitory role for nutritional antioxidants and serum paraoxonase. Clin Chem Lab Med 37: 777-787, 1999.

KAY CD, HOLUB BJ: The effect of wild blueberry (Vaccinium angustifolium) consumption on postprandial serum antioxidant status in human subjects. Brit J Nutr 88: 389-397, 2002.

KHKONEN MP, HEINONEN M: Antioxidant activity of anthocyanins and their Aaglycons. J Agric Food Chem 51: 628-633, 2003.

KÜHNAU J: The flavonoids: a class of semi-essential food components: their role in human nutrition. World Rev Nutr Diet 24: 117-120, 1976.

LOTTO S, FREI B: The increase in human plasma antioxidant capacity after apple consumption is due to the metabolic effect of fructosio on urate, non apple derived antioxidant flavonoids. Free Radic Biol Med 37: 251-258, 2004.

MADSEN KL, ARIANO D, FEDORAK RN: Insulin dawn regulates diabetic-enhanced intestinal glucose transport rapidly in ileum and slowly in jejunum. Can J Physiol Pharmacol 74: 1294-1301, 1996.

MAZZA G, KAY CD, COTTRELL T, HOLUB BJ: Absorption of anthocyanins from blueberries and serum antioxidant status in human subjects. J Agric Food Chem 50: 7731-7737, 2002.

MORAN JF, KLUCAS RV, GRAYER RJ, ABIAN J, BECANA M: Complexes of iron with phenolic compounds from soybean nodules and other legume tissues: prooxidant and antioxidant properties. Free Radic Biol Med 22: 861-870, 1997.

OBI FO, USENU IA, OSAYANDE JO: Prevention of carbon tetrachloride-induced hepatotoxicity in the rat by $H$. rosasinensis anthocyanin extract administered in ethanol. Toxicology 131: 93-98, 1998.

OHTA Y, NISHIDA K, SASAKI E, KONGO M, ISHIGURO I: Attenuation of disrupted hepatic active oxygen metabolism with the recovery of acute liver injury in rats intoxicated with carbon tetrachloride. Res Commun Mol Pathol Pharmacol 95: 191-207, 1997.

OU B, HAMPSCH-WOODILL M, PRIOR RL: Development and validation of an improved oxygen radical absorbance capacity assay using fluorescein as the fluorescent probe. J Agric Food Chem 49: 4619-4626, 2001.

OZTURK F, UCAR M, OZTURK IC, VARDI N, BATCIOGLU K: Carbon tetrachloride- induced nephrotoxicity and protective effect of betaine in Sprague-Dawley rats. Urology 62: 353-356, 2003.

PRIOR RL: Absorption and methabolism of anthocyanins: potential health effects. In: Phytochemicals: Mechanisms of Action. MESKIN MS, BIDLACK WR, DAVIES AJ, LEWIS DS, RANDOLPH RK (eds), CRC Press, 2004, pp 1-20.

PRIOR RL, WU XL: Anthocyanins: Structural characteristics that result in unique metabolic patterns and biological activities. Free Radic Res 40: 1014-1028, 2006.

PRIOR RL, XIANLI W, SCHAICH K: Standardised methods for the determination of antioxidant capacity and phenolics in foods and dietary supplements. J Agric Food Chem 53: 4290-4302, 2005.

REINKE LA, LAI EK, MCCAY PB: Ethanol feeding stimulates trichloromethyl radical formation from carbon tetrachloride in liver. Xenobiotica 18: 1311-1318, 1988. 
SALONEN JT, NYYSSONEN K, SALONIN R, LAKKA HM, KAIKKONEN J, PORKKALA-SARATATHO E, VOUTILAINEN S, LAKKA TA, RISSANEN T, LESKINEN L, TUOMAINEN TP, VALKONEN VP, VOUTILAINEN S, POULSEN HE: Antioxidant supplementation in atherosclerosis prevention (ASAP) study: a randomized trial of the effects of vitamin $\mathrm{E}$ and $\mathrm{C}$ on 3-year progression of carotid atherosclerosis. J Int Med 248: 377-386, 2000.

SANNINO A, MADAGHIELE M, LIONETTO MG, SCHETTINO T, MAFFEZZOLI A: A cellulose-based hydrogel as a potential bulking agent for hypocaloric diets: an in vitro biocompatibility study on rat intestine. $J$ Appl Polym Sci 102: 1524-1530, 2006.

SINGLETARY KW, JUNG KJ, GIUSTI M: Anthocyanin-rich grape extract blocks breast cell DNA damage. $J$ Med Food 10: 244-251, 2007.

SMITH PL: Methods for evaluating intestinal permeability and metabolism in vitro. Pharm Biotechnol 8: 13-34, 1996.

SZYMONIK-LESIUK S, CZECHOWSKA G, STRYJECKA-ZIMMER M, SLOMKA M, MADRO A, CELINSKI K, WIELOSZ M: Catalase, superoxide dismutase, and glutathione peroxidase activities in various rat tissues after carbon tetrachloride intoxication. J Hepatobiliary Pancreat Surg 10: 309-315, 2003.

TIRKEY N, PILKHWAL S, KUHAD A, CHOPRA K: Hesperidin, a citrus bioflavonoid, decreases the oxidative stress produced by carbon tetrachloride in rat liver and kidney. BMC Pharmacol 5: 2, 2005.

VENDEMIALE G, GRATTAGLIANO I, ALTOMARE E: An update on the role of free radicals and antioxidant defence in human disease. Int J Clin Lab Res 29: 49-55, 1999.

WHITTLE BJR, STEEL G: Evaluation of the protection of rat gastric mucosa by a prostaglandin analogue using cellular enzyme marker and histological techniques. Gastroenterology 88: 315-327, 2002.

WILLETT WC: Diet and cancer: one view at the start of the millennium. Cancer Epidemiol Biomarkers Prev 10: 3-8, 2001.

WROBLEWSKI F, LA DUE J: Lactic dehydrogenase activity in blood. Proc Soc Exp Biol Med 90: 210-213, 1955.

WU X, CAO G, PRIOR RL: Absorption and metabolism of anthocyanins in human subjects following consumption of elderberry or blueberry. J Nutr 132: 1865-1871, 2002.

YILMAZ Y, TOLEDO RT: Oxygen radical absorbance capacities of grape/wine industry byproducts and effect of solvent type on extraction of grape seed polyphenols. J Food Comp Analysis 19: 41-48, 2006.

ZENEBE W, PECHÁŇOVÁ O, ANDRIANTSITOHAINA R: Red wine polyphenols induce vasorelaxation by increased nitric oxide bioactivity. Physiol Res 52: 425-432, 2003. 